\title{
Roster of the American Society for Clinical Investigation
}

1959

\section{ACTIVE MEMBERS}

Abelmann, Walter $H$. Heart Station

Boston City Hospital

Boston 18, Massachusetts

Ahrens, Edward H., JR. The Rockefeller Institute

66th Street and York Avenue New York 21, New York

Albright, Edwin C.

University Hospitals

Department of Medicine

1300 University Avenue

Madison 6, Wisconsin

Albrink, Margaret J.

Yale University School of Medicine

333 Cedar Street

New Haven 11, Connecticut

Almy, Thomas P.

Second (Cornell) Medical

Division

Bellevue Hospital

New York 16, New York

ANDREs, Reubin

Baltimore City Hospitals

4940 Eastern Avenue

Baltimore 24, Maryland

Asper, Samuel P., Jr.

The Johns Hopkins Hospital

Baltimore 5, Maryland

Atrins, Elisha

Department of Internal Medicine

333 Cedar Street

New Haven 11, Connecticut

Austrian, Robert

A Building

Kings County Hospital

Brooklyn 3, New York

Baldwin, David S.

20 East 68th Street

New York 21, New York
BANG, Frederik B.

School of Hygiene

615 N. Wolfe Street

Baltimore 5, Maryland

Barclay, William R.

University of Chicago

950 East 59th Street

Chicago 37, Illinois

Barnett, Henry Lewis

Albert Einstein College of Medicine

Eastchester Road \& Morris

Park Avenue

New York 61, New York

Bartter, Frederic C.

Clinical Center NHI

National Institutes of Health

Bethesda 14, Maryland

BAtes, D. V.

Department of Medicine

Royal Victoria Hospital

Montreal 2, Canada

Bearn, Alexander G.

The Rockefeller Institute for Medical Research

66th Street \& York Avenue

New York 21, New York

Beck, Johannes C.

McGill University Clinic

Royal Victoria Hospital

Montreal, P. Q., Canada

Beck, William S.

Hematology Research

Laboratory

Massachusetts General Hospital

Boston 14, Massachusetts

BENNETT, IVAN

Department of Pathology

The Johns Hopkins Hospital

Baltimore 5, Maryland
Berliner, Robert William

4317 Elm Street

Chevy Chase, Maryland

Berne, Robert M.

Department of Physiology

School of Medicine

Western Reserve University

Cleveland 6, Ohio

Berson, Solomon A.

159 Yale Street

Roslyn Heights, New York

Beutler, Ernest

Department of Medicine

University of Chicago

950 East 59th Street

Chicago 37, Illinois

Bierman, Howard R.

9730 Wilshire Boulevard

Beverly Hills, California

Blake, William D.

Department of Physiology

University of Oregon Medical School

Portland 1, Oregon

Block, Matthew

University of Colorado

Department of Medicine

4200 East Ninth Avenue

Denver 20, Colorado

Bloom, Walter L.

Piedmont Hospital

1958 Peachtree Road, N.W.

Atlanta 9, Georgia

Blount, S. Gilbert, Jr.

Head, Division of Cardiology

University of Colorado Medical School

Denver 20, Colorado 


Bollet, AlFRED JAY
Department of Preventive
Medicine
University of Virginia School of
Medicine
Charlottesville, Virginia
Bondy, PHILIP K.
Department of Internal
Medicine
Yale University School of
Medicine
New Haven 11, Connecticut
BongiovanNi, ALFred M.
Children's Hospital of
Philadelphia
1740 Bainbridge Street
Philadelphia 46, Pennsylvania

Braude, A. I.

Department of Medicine

University of Pittsburgh

Pittsburgh, Pennsylvania

Bricker, Neal S.

Washington University

School of Medicine

St. Louis 10, Missouri

Brodsky, William A.

University of Louisville School of Medicine

Department of Pediatrics

101 West Chestnut Street

Louisville, Kentucky

Brody, Daniel Anthony

858 Madison Avenue

Memphis 3, Tennessee

Brown, G. Malcolm

E Therington Hall

Stuart Street

Kingston, Ontario, Canada

Brown, Hakold

Veterans Administration Hospital

Salt Lake City, Utah

Brown, Ivan W., Jr.

Department of Surgery

Duke University Hospital

Durham, North Carolina

Bruce, Robert A.

Department of Medicine

University of Washington

Seattle 5, Washington
Brust, Albert A., Jr.

Kettering Laboratory

University of Cincinnati

Eden Avenue

Cincinnati 19, Ohio

Bunn, Paul A.

State University of New York

Upstate Medical Center

Department of Medicine

University Hospital of the Good Shepherd

Syracuse 10, New York

Burrows, Belton A.

Evans Memorial Hospital

65 East Newton Street

Boston 18, Massachusetts

Calder, Abbie Knowlton

110 East 78th Street

New York 21, New York

Cameron, Douglas G.

Department of Medicine

Montreal General Hospital

Montreal, Canada

Carroll, Douglas, Jr.

Brooklandville, Maryland

Cartwright, G. E.

University of Utah School of Medicine

Department of Internal

Medicine

Salt Lake General Hospital

175 East 21st South Street

Salt Lake City 5, Utah

Chalmers, Thomas C.

33 Moon Hill Road

Lexington 73, Massachusetts

Chaplin, Hugh, JR.

Department of Preventive Medicine

Washington University School of Medicine

Euclid \& Kingshighway

St. Louis 10, Missouri

Chapman, Carleton B.

Department of Internal Medicine

Southwestern Medical School of The University of Texas 5323 Harry Hines Boulevard

Dallas, 35, Texas

Cherniack, R. M.

Medical Buildings

Bannatyne \& Emily

Winnipeg 3, Manitoba, Canada
Chernoff, A. I.

University of Tennessee

Memorial Research Center and Hospital

Knoxville, Tennessee

Chinard, Francis P.

The Johns Hopkins School of Medicine

Department of Physiological Chemistry

710 N. Washington Street

Baltimore 5, Maryland

Christy, Nicholas P.

Department of Medicine

P \& S-Columbia University

620 West 168th Street

New York 32, New York

Clark, John Kapp

837 Gates Building

Hospital of the University of Pennsylvania

36th and Spruce Streets

Philadelphia 4, Pennsylvania

Cluff, Leighton E.

The Johns Hopkins Hospital

Baltimore 5, Maryland

Conley, C. Lockard

The Johns Hopkins Hospital

Baltimore 5, Maryland

Conn, Hadley L., Jr.

905 Maloney Clinic Building

University Hospital

34th \& Spruce Streets

Philadelphia 4, Pennsylvania

Cooke, Robert E.

Department of Pediatrics

The Johns Hopkins Hospital

Baltimore 5, Maryland

Cotzias, George C.

Head, Physiology Division

Medical Research Center

Brookhaven National Laboratory

Upton, Long Island, New York

Crawhord, John D.

Children's Medical Service

Massachusettes General Hospital

Fruit Street

Boston 14, Massachusetts 
Crispell, Kenneth R.

New York Medical College

Department of Internal Medicine

106th Street at Fifth Avenue

New York 29, New York

Cronkite, Eugene P.

Brookhaven National Laboratory Medical Department

Head, Experimental Pathology

Upton, Long Island, New York

Crosby, Lt. Col. William H.

Department of Hematology

Walter Reed Army Institute of Research

Walter Reed Army Medical Center

Washington 12, D. C.

Crosley, Archer P., Jr.

837 Gates Building

Hospital of the University of Pennsylvania

36th \& Spruce Streets

Philadelphia 4, Pennsylvania

Crumpton, Charles W.

Wisconsin General Hospital

Department of Medicine

1300 University Avenue

Madison 6, Wisconsin

Cummings, Martin M.

Department of Microbiology

University of Oklahoma Medical Center

Oklahoma City, Oklahoma

Curran, George L.

Department of Internal Medicine

St. Louis University School of Medicine

1325 South Grand Boulevard

St. Louis 4, Missouri

Danowski, T. S.

930-936 Health Professional Building

University of Pittsburgh School of Medicine

Pittsburgh 13, Pennsylvania

Daughaday, William $\mathrm{H}$.

Department of Medicine

Washington University School

of Medicine

St. Louis 10, Missouri
DAVEnPort, Fred M.

School of Public Health

University of Michigan

Ann Arbor, Michigan

Deiss, William P.

Indiana University School of Medicine

1100 W. Michigan Street

Indianapolis 7, Indiana

Deming, Quentin B.

Columbia University Research Service

Goldwater Memorial Hospital

Welfare Island

New York 17, New York

DiPalma, Joseph R.

Hahnemann Medical School

235 North 15th Street

Philadelphia 2, Pennyslvania

Dixon, Frank J., JR.

Department of Pathology

School of Medicine

University of Pittsburgh

Pittsburgh 13, Pennsylvania

DuBois, Arthur B.

University of Pennsylvania

Graduate School of Medicine

Departments of Physiology and Pharmacology

Philadelphia 4, Pennsylvania

Dustan, Harriet P.

Research Division

Cleveland Clinic Foundation

Cleveland 6, Ohio

Eberlein, Walter R.

Children's Hospital

University of Pennsylvania

1740 Bainbridge Street

Philadelphia 46, Pennsylvania

Eckel, Robert E.

School of Medicine

Western Reserve University

Cleveland 6, Ohio

Edelman, I. S.

University of California Medical Center

San Francisco 22, California

Eder, How ard A.

Albert Einstein College of Medicine

Eastchester Rd. \& Morris Park Avenue

New York 61, New York
Eisen, Herman N.

Department of Medicine

Division of Dermatology

School of Medicine

Washington University

600 S. Kingshighway

St. Louis 10, Missouri

EldRIDGe, F. L.

Stanford University School of Medicine

304 Pasteur Drive

Palo Alto, California

Eliel, Leonard P.

Oklahoma Medical Research Institute

825 N. E. Thirteenth Street

Oklahoma City 4, Oklahoma

Engstrom, W. W.

Marquette University

School of Medicine

Milwaukee County General Hospital

Department of Medicine

Milwaukee 13, Wisconsin

Epstein, Franklin H.

Department of Medicine

Yale University School of Medicine

333 Cedar Street

New Haven 11, Connecticut

Erslev, Allan J.

The Cardeza Foundation

Jefferson Medical College

Philadelphia, Pennsylvania

Fajans, Stefan S.

University Hospital

Ann Arbor, Michigan

FARber, SaUl J.

New York University College

of Medicine

550 First Avenue

New York 16, New York

FERrer, M. Irene

10 East 66th Street

New York 21, New York

Finch, Clement A.

Department of Medicine

AA-512 University Hospital

University of Washington

Seattle 5, Washington 
Finch, Stuart C.

Department of Internal Medicine

Yale University School of Medicine

New Haven 11, Connecticut

Fischel, Edward E.

Director, Department of Medicine

The Bronx Hospital

New York 56, New York

Fishman, Alfred P.

541 East 20th Street

New York 10, New York

Fitzpatrick, Thomas B.

3181 S.W. Sam Jackson

Boulevard

Portland 1, Oregon

Fletcher, Anthony P. 5414 Delmar Boulevard

St. Louis 12, Missouri

Forsham, Peter H. University of California Hospital San Francisco 22, California

Forster, Robert E.

721 Millbrook Lane

Haverford, Pennsylvania

Fowler, Ward S.

Section on Physiology

Mayo Clinic

Rochester, Minnesota

Frantz, Ivan D., Jr.

Department of Medicine

University of Minnesota

Hospitals

Minneapolis 14, Minnesota

Fredrickson, Donald S.

Section on Metabolism

National Heart Institute

Bethesda 14, Maryland

Frein kel, Norbert

Thorndike Memorial Laboratory

Boston City Hospital

Boston 18, Massachusetts

Fritts, Harry W., Jr.

Cardiopulmonary Laboratory (C-6)

Bellevue Hospital

462 First Avenue

New York 16, New York
Gabuzda, George J.

Cleveland Metropolitan General Hospital

3395 Scranton Road

Cleveland 9, Ohio

Gardner, Frank H.

Peter Bent Brigham Hospital

Boston 15, Massachusetts

Ginsberg, Harold S.

Department of Preventive Medicine

School of Medicine

Western Reserve University

Cleveland 6, Ohio

Gitlin, David

Harvard Medical School

Department of Pediatrics

Children's Hospital

300 Longwood Avenue

Boston, Massachusetts

Glaser, Robert J.

Office of the Dean

University of Colorado Medical Center

4200 East Ninth Avenue

Denver 20, Colorado

Goldthwait, David A.

Department of Biochemistry

Western Reserve University

School of Medicine

2109 Adelbert Road

Cleveland 6, Ohio

Good, Robert A.

608 Oak Street, S. E.

Minneapolis 14, Minnesota

Goodyer, Allan V. N.

Yale University School of Medicine

333 Cedar Street

New Haven 11, Connecticut

Gordan, Gilbert S., Jr.

University of California Hospital

San Francisco 22, California

Gordon, Irving

Department of Medical Microbiology

School of Medicine

University of Southern California

University Park

Los Angeles 7, California

Gordon, Robert S., Jr.

3915 Prospect Street

Kensington, Maryland
Gorlin, Richard

Peter Bent Brigham Hospital

721 Huntington Avenue

Boston 15, Massachusetts

Gottschalk, Carl W.

Department of Medicine

University of North Carolina

Chapel Hill, North Carolina

Grant, Robert P.

National Heart Institute

Bethesda 14, Maryland

Gray, Frank D., JR.

Yale University School of Medicine

Department of Internal Medicine

New Haven 11, Connecticut

Grayston, J. Thomas

U. S. Naval Medical Research Unit

APO 63

San Francisco, California

Greer, Monte A.

University of Oregon Medical School

Department of Medicine

Portland 1, Oregon

Grob, David

Maimonides Hospital

4802 Tenth Avenue

Brooklyn 19, New York

Grossman, Morton I.

Veterans Administration Center

Los Angeles 25, California

Grumbach, Melvin M.

Babies Hospital

Presbyterian Hospital

3975 Broadway

New York 32, New York

Hall, Wendell $\mathrm{H}$.

Chief, Laboratory Service

Veterans Administration

Hospital

54th St. \& 48th Avenue South Minneapolis 17, Minnesota

Hamerman, David

Albert Einstein College of Medicine

Department of Medicine

Eastchester Rd. \& Morris Park Avenue

New York 61, New York 
Hamolsky, Milton W.

Beth Israel Hospital

330 Brookline Avenue

Boston 15, Massachusetts

Harrington, William J.

Washington University

School of Medicine

600 South Kingshighway

St. Louis 10, Missouri

HARRIS, JOHN W.

Cleveland Metropolitan General Hospital

3395 Scranton Road

Cleveland 9, Ohio

Harvey, Rejane M.

10 East 66th Street

New York 21, New York

Havel, Richard J.

University of California Medical Center

San Francisco 22, California

Hellems, HaR PER K.

319 Moross

Grosse Pointe Farms 36,

Michigan

Hellman, Leon

334 West 245th Street

New York 71, New York

Henneman, Philip $\mathrm{H}$.

Seton Hall Medical School

Jersey City 4, New Jersey

Heyman, Albert

Duke Hospital

Box 3203

Durham, North Carolina

Hiatt, Howard H.

Beth Israel Hospital

330 Brookline Avenue

Boston 15, Massachusetts

Hickam, John Bamber

Indiana University Medical Center

1100 West Michigan Street

Indianapolis 2, Indiana

Hills, A. Gorman

Jackson Memorial Hospital

Miami 36, Florida

Hirsch, James G.

Rockefeller Institute for Medical Research

66th Street \& York Avenue

New York 21, New York
Hirsch, Jules

Rockefeller Institute for

Medical Research

66th Street \& York Avenue

New York 21, New York

Hoffman, Martin M.

1374 Sherbrooke St. W. No. $16 \mathrm{~A}$

Montreal, Quebec, Canada

Hollander, Walter, Jr.

531 Dogwood Drive

Chapel Hill, North Carolina

HOllingsworth, J. W.

Atomic Bomb Casualty

Commission

MCAF Navy 955

c/o FPO

San Francisco, California

Horrigan, Daniel L.

University Hospitals

2065 Adelbert Road

Cleveland 6, Ohio

Howe, Calderon

College of Physicians \&

Surgeons

630 West 168th Street

New York 32, New York

Huckabee, William E.

Massachusetts Memorial

Hospital

750 Harrison Avenue

Boston 18, Massachusetts

Huff, Rex L.

416 Central Avenue

Elizabethtown, Kentucky

Hultgren, Herbert N.

Cardiology Laboratory

Stanford University School of Medicine

300 Pasteur Drive

Palo Alto, California

Hume, David M.

Department of Surgery

Medical College of Virginia

Hospital

1200 East Broad Street

Richmond 19, Virginia

INGBAR, Sidney H.

46 Kingswood Road

Auburndale 66, Massachusetts

ISSELBACHER, KURT J.

Massachusetts General Hospital

Boston 14, Massachusetts
Jackson, Dudley P.

The Johns Hopkins University

Medical School

Baltimore 5, Maryland

Jackson, George Gee

University of Illinois College of Medicine

Department of Medicine

840 South Wood Street

Chicago 12, Illinois

James, G. Watson, III

Laboratory for Clinical Investigation

604 Clinic Building

Medical College of Virginia

Richmond, Virginia

Jandl, James Harriman

Thorndike Memorial Laboratory

Boston City Hospital

Boston 18, Massachusetts

Janowitz, Henry D.

The Mount Sinai Hospital

One East 100th Street

New York 29, New York

JAwetz, ERnest

University of California

Medical Center

San Francisco 22, California

Jensen, Wallace N.

534 Greenhurst Drive

Mount Lebanon, Pennsylvania

Johnson, Alan J.

Department of Medicine

New York University

550 First Avenue

New York 16, New York

Jordan, William S.

Department of Preventive Medicine

University School of Medicine

Charlottesville, Virginia

Kass, Edward H.

Boston City Hospital

Harvard Medical School

Boston 18, Massachusetts

Keeney, Edmund L.

Scripps Clinic \& Research

Foundation

476 Prospect Street

La Jolla, California

Kety, Seymour S.

5613 Warwick Place

Chevy Chase 15, Maryland 
Kilbourne, Edwin D.

Department of Public Health and Preventive Medicine

Cornell University Medical College

1300 York Avenue

New York 21, New York

Kirby, William M. M.

Department of Medicine

University of Washington

School of Medicine

Seattle 5, Washington

Kleeman, Charles R.

Department of Medicine

V. A. Center \& Hospital

Wilshire \& Sawtelle Blvds.

Los Angeles 25, California

Knight, Vernon

Laboratory of Clinical Investigation

National Institute of Allergy \& Infectious Diseases

National Institutes of Health

Bethesda 14, Maryland

Koch-Weser, Dieter

2065 Adelbert Road

Cleveland 6, Ohio

Korey, Saul R.

Department of Medicine (Neurology)

Albert Einstein College of Medicine

New York 61, New York

Kretchmer, Norman

Department of Pediatrics

Stanford University

School of Medicine

Palo Alto, California

Kuhns, William J.

Central Blood Bank of

Pittsburgh

University of Pittsburgh Medical Center

Pittsburgh 13, Pennsylvania

Kunkel, Henry George

Rockefeller Institute for Medical Research

66th Street \& York Avenue

New York 21, New York

KyLE, L. H.

Georgetown Hospital

Washington 7, D. C.
Labiy, Daniel H.

University of Oregon Medical School

Department of Medicine

3181 S. W. Sam Jackson Park Road

Portland 1, Oregon

Lambertsen, C. J.

217 Glenn Road

Ardmore, Pennsylvania

LANDAC, Richard L.

The University of Chicago

Department of Medicine

Chicago 37, Illinois

LaRAGH, John H.

Presbyterian Hospital

622 West 168th Street

New York 32, New York

Larson, Daniel L.

622 West 168th Street

New York 32, New York

Lasagna, Louis

Sta. 915

The Johns Hopkins Hospital

Baltimore 5. Maryland

Lathem, Willoughby

Department of Medicine

University of Pittsburgh

School of Medicine

Pittsburgh 13, Pennsylvania

Lawrence, H. Sherwood

N. Y. U.-Bellevue Medical Center

Department of Medicine

550 First Avenue

New York 16, New York

Leaf, Alexander

Massachusetts General Hospital

Boston 14, Massachusetts

Lepper, Mark H.

327 Hampton Place

Hinsdale, Illinois

Levenson, Stanley M.

Department of Surgical Metabolism and Physiology

WRAIR WRAMC

Washington 12, D. C.

Levitt, Marvin F.

4 East 89th Street

New York 28, New York
Lewis, Jessica $H$.

Department of Medicine

University of Pittsburgh

Pittsburgh 13, Pennsylvania

Liddle, Grant Winder

Department of Medicine

Vanderbilt University School of

Medicine

Nashville 5, Tennessee

LIPSKY, S. R.

Department of Medicine

Yale University

School of Medicine

New Haven 11, Connecticut

London, Irving Myer

Albert Einstein College of

Medicine

New York 61, New York

Lowe, Charles U.

Children's Hospital

219 Bryant Street

Buffalo 22, New York

Lozner, Eugene L.

University Hospital of the Good Shepherd

Syracuse 10, New York

Lynn, William S., Jr.

Duke University Medical Center

Durham, North Carolina

Maddock, William O.

Doctors Clinic

423 D Street

Anchorage, Alaska

Marks, Paul A.

Department of Medicine

College of Physicians \&

Surgeons

630 W. 168th Street

New York 32, New York

Martin, Samuel P.

J. Hillis Miller Medical Center

University of Florida

Gainesville, Florida

McKusick, Victor A.

The Johns Hopkins Hospital

Baltimore 5, Maryland

Meads, Manson

The Bowman-Gray School of Medicine

Winston-Salem 7, North

Carolina 
Mendeloff, Albert I.

Sinai Hospital

Monument Street at Rutland Avenue

Baltimore 5, Maryland

Merrill, John P.

Peter Bent Brigham Hospital

721 Huntington Avenue

Boston 15, Massachusetts

Metcoff, JACK

Michael Reese Hospital

Department of Pediatrics

29th Street \& Ellis Avenue

Chicago 16, Illinois

Michael, Max, Jr.

Duval Medical Center

2000 Jefferson Street

Jacksonville, Florida

Middlebrook, Gardner

National Jewish Hospital

3800 East Colfax Avenue

Denver 6, Colorado

Morgan, Herbert R.

Department of Bacteriology

Strong Memorial Hospital

260 Crittenden Boulevard

Rochester 7, New York

Motulsky, Arno G.

Department of Medicine

University of Washington

School of Medicine

Seattle 5, Washington

Mudge, Gilbert $\mathrm{H}$.

Department of Pharmacology \& Experimental Therapeutics

The Johns Hopkins School of Medicine

710 N. Washington Street

Baltimore 5, Maryland

Mueller, John F.

Department of Medicine

Cincinnati General Hospital

Cincinnati 29, Ohio

Muirhead, E. E.

College of Medicine

Wayne State University

Detroit 7, Michigan

Nelson, Don Harry

721 Huntington Avenue

Boston 15, Massachusetts
Nelson, Robert A., Jr.

Department of Medicine

Yale School of Medicine

310 Cedar Street

New Haven 11, Connecticut

Olson, Robert E.

Graduate School of Public Health

Department of Biochemistry \& Nutrition

University of Pittsburgh

Pittsburgh 13, Pennsylvania

OrLOFF, JACK

National Heart Institute

National Institutes of Health

Bethesda 14, Maryland

Osserman, Elliott F.

150 Columbus Avenue

Closter, New Jersey

PAPper, E. M.

622 West 168th Street

New York 32, New York

Park, Charles $R$.

Vanderbilt University School of Medicine

Department of Physiology

Nashville 5, Tennessee

Pechet, Maurice M.

Lowell House-1-34

Harvard University

Cambridge, Massachusetts

Peterson, Lysle $H$.

University of Pennsylvania

School of Medicine

Department of Physiology

Philadelphia 4, Pennsylvania

Peterson, Ralph E.

Cornell University Medical School

Department of Medicine

525 East 68th Street

New York 21, New York

Preedy, John R. K.

Emory University

School of Medicine

69 Butler Street, S. E.

Atlanta 3, Georgia

Price, Henry L.

Room 811, Gates Pavilion

Hospital of the University of Pennsylvania

Philadelphia 4, Pennsylvania
Pullman, Theodore N.

University of Chicago

Department of Medicine

Billings Hospital

Chicago 37, Illinois

Raben, Maurice S.

703 Chestnut Street

Waban, Massachusetts

RALL, J. E.

National Institute of Arthritis \& Metabolic Diseases

National Institutes of Health

Bethesda 14, Maryland

Ranney, Helen M.

Presbyterian Hospital

622 West 168th Street

New York 32, New York

Ratnoff, Oscar D.

University Hospitals

Cleveland 6, Ohio

RAy, C. ThORPE

University of Missouri

Medical Center

Columbia, Missouri

Recant, LiLlian

Washington University

Department of Preventive Medicine

507 South Euclid

St. Louis, Missouri

Reiser, Morton F.

735 Kappock Street

New York 63, New York

Relman, Arnold S.

Evans Memorial Hospital

65 East Newton Street

Boston 18, Massachusetts

Renold, Albert Ernest

Baker Clinic Research

Laboratory

New England Deaconess

Hospital

185 Pilgrim Road

Boston 15, Massachusetts

Reynolds, Telfer B.

1200 North State Street

Los Angeles 33, California

Robbins, Frederick C.

Department of Pediatrics \& Contagious Diseases

Cleveland Metropolitan Gen-

eral Hospital

3395 Scranton Road

Cleveland 9, Ohio 
Robbins, Jacob

National Institute of Arthritis

\& Metabolic Diseases

Bethesda 14, Maryland

Roberts, Kathleen E.

USPHS Hospital

15th \& Lake Streets

San Francisco, California

Robins, ELI

Department of Psychiatry \& Neurology

Washington University School of Medicine

4580 Scott Avenue

St. Louis 10, Missouri

Rogers, David Elliott

Department of Medicine

Vanderbilt University School of Medicine

Nashville 5, Tennessee

ROSENBERG, ISADORE N.

12 Whittlesey Road

Newton Centre 59,

Massachusetts

Rosenman, Ray H. 2245 Post Street

San Francisco 15, California

Rowe, Wallace P.

National Institutes of Health

Bethesda 14, Maryland

Sandberg, Avery A.

Roswell Park Memorial Institute

Buffalo 3, New York

Sarnoff, Stanley J.

Chief, Laboratory of Cardiovascular Physiology

NHI Bldg. 10 Room 6D-20

National Institutes of Health

Bethesda 14, Maryland

Sborov, Victor M.

945 Middlefield Road

Redwood City, California

Schein berg, I. Herbert

Albert Einstein College of Medicine

Eastchester Road \& Morris Park Avenue

Bronx 61, New York

Schilling, Robert F.

University Hospitals

1300 University Avenue

Madison 6, Wisconsin
Schmid, Rudi

Thorndike Memorial Laboratory

Boston City Hospital

Boston 18, Massachusetts

Schnabel, Truman Gross, Jr. 209 Woodside Avenue

Narberth, Pennsylvania

Schreiner, George E.

Department of Medicine

Georgetown University Hospital

Washington 7, D. C.

Schwartz, Irving L.

Medical Research Center

Brookhaven National

Laboratory

Upton, Long Island, New York

Schwartz, Samuel

Department of Medicine

University of Minnesota

School of Medicine

Minneapolis 14, Minnesota

Schwartz, Theodore B.

Director, Section on Endocrinology and Metabolism

Presbyterian St. Luke's Hospital

Chicago 12, Illinois

Schwartz, William B.

New England Center Hospital

171 Harrison Avenue

Boston 11, Massachusetts

Scribner, Belding $\mathrm{H}$.

Department of Medicine

University of Washington

Seattle 5, Washington

Segaloff, Albert

Division of Endocrinology

Alton Ochsner Medical Foundation

1520 Jefferson Highway

New Orleans 21, Louisiana

Seldin, Donald W.

Department of Internal Medicine

University of Texas

Southwestern Medical School

5323 Harry Hines Blvd.

Dallas 19, Texas

Seligson, David

Yale-New Haven Medical Center

New Haven 4, Connecticut
Shank, R. E.

Washington University

Department of Preventive Medicine

507 South Euclid

St. Louis, Missouri

Shepard, Richard $H$.

212 East Gittings Avenue

Baltimore 12, Maryland

Sherry, Sol

Department of Internal Medicine

Washington University School of Medicine

600 South Kingshighway

St. Louis 10, Missouri

Sieker, Herbert O.

Department of Medicine

Duke University School of Medicine

Durham, North Carolina

Siperstein, Marvin David

University of Texas Southwestern Medical School

5323 Harry Hines Boulevard

Dallas, Texas

Sjoerdsma, Albert

National Heart Institute

Bethesda 14, Maryland

Sleisenger, Marvin $H$.

The New York Hospital

525 East 68th Street

New York 21, New York

Smith, Richard $T$.

The University of Florida

Department of Pediatrics

M-316 Medical Sciences Bldg.

J. Hillis Miller Health Center Gainesville, Florida

Sonnenberg, Martin

444 East 68th Street

New York 21, New York

Spaet, Theodore $H$.

Montefiore Hospital

210th St. \& Bainbridge Avenue

New York 67, New York

Stamler, Jeremiah

Heart Disease Control Program

Chicago Board of Health

54 West Hubbard Street

Chicago 10, Illinois

Stanbury, John B.

Massachusetts General Hospital

Fruit Street

Boston 14, Massachusetts 
Stanley, Malcolm M.

University of Louisville School of Medicine

323 E. Chestnut Street

Louisville 2, Kentucky

Stead, William W.

J. Hillis Miller Health Center

Gainesville, Florida

Stefanini, Mario

Department of Medical Research

St. Elizabeth Hospital

736 Cambridge Street

Brighton 35, Massachusetts

Sterling, Kenneth

Columbia-Presbyterian Medical Center

722 West 168th Street

New York 32, New York

Stetson, Chandler A., JR.

Department of Pathology

N. Y. U.-Bellevue Medical Center

550 First Avenue

New York 16, New York

Stohlman, Frederick, Jr.

National Institutes of Health

Bethesda 14, Maryland

Stollerman, Gene H.

Department of Medicine

Northwestern University Medical School

303 East Chicago Avenue

Chicago 11, Illinois

Swan, Roy C., JR.

Department of Anatomy

Cornell Medical College

1300 York Avenue

New York 21, New York

Swisher, Scott N.

260 Crittenden Blvd.

Rochester 20, New York

TAgGart, JoHn V.

Columbia University College of

Physicians \& Surgeons

Department of Medicine

Enzyme Laboratory

630 West 168th Street

New York 32, New York

Talmage, David W.

Department of Medicine

Colorado Medical Center

4200 East 9th Avenue

Denver 20, Colorado
TAMM, IGOR

Rockefeller Institute for Medical Research

66th Street \& York Avenue

New York 21, New York

Tenney, S. M.

Department of Physiology

Dartmouth Medical School

Hanover, New Hampshire

Thomas, Edward Donnall

The Mary Imogene Bassett

Hospital

Cooperstown, New York

Thompson, David D.

525 East 68th Street

Room F-211

New York 21, New York

Tobian, Louis, JR.

549 Otis Avenue

St. Paul 4, Minnesota

Tyler, Frank $\mathrm{H}$.

Department of Internal Medicine

University of Utah

175 East 21st South Street

Salt Lake City 15, Utah

Valentine, William Newton

Department of Medicine

University of California Medical School

Los Angeles 24, California

VAllee, Bert L.

Trace Metals Laboratory

Peter Bent Brigham Hospital

721 Huntington Avenue

Boston 15, Massachusetts

VANDERLAan, Willard P. 8275 La Jolla Shores Drive

La Jolla, California

Vaughan, JoHN H.

The University of Rochester

Medical Center

260 Crittenden Boulevard

Rochester 20, New York

VolWILER, WADE

University of Washington

Seattle 5, Washington

WAgner, ROBERT R.

Department of Microbiology

The Johns Hopkins University

School of Medicine

Baltimore 5, Maryland
WanNamaker, LeWIS W.

Department of Pediatrics

University of Minnesota

The Medical School

Minneapolis 14, Minnesota

WARREN, JAMES VAUGHN

Department of Internal Medicine

University of Texas

Medical School

Galveston, Texas

Waterhouse, Christine

Strong Memorial Hospital

260 Crittenden Boulevard

Rochester 20, New York

Wedgwood, Ralph J. P.

Western Reserve University

2065 Adelbert Road

Cleveland 6, Ohio

Wessler, Stanford

71 Fresh Pond Lane

Cambridge 38, Massachusetts

Weston, Raymond E.

803 North Roxbury Drive

Beverly Hills, California

Wisseman, Charles L., Jr.

Department of Microbiology

University of Maryland Medical School

Baltimore 1, Maryland

Wright, Claude-Starr

Department of Medicine

Medical College of Georgia

Augusta, Georgia

WyngaARden, James B.

Box 3009

Duke University School of Medicine

Department of Medicine

Durham, North Carolina

ZieRLER, KenNeTH L.

The Johns Hopkins Hospital

601 North Broadway

Baltimore 5, Maryland

Zimmerman, Hyman J.

846 North Kenilworth

Oak Park, Illinois

ZiNSSER, HARRY F., JR.

University of Pennsylvania

Hospital

36th \& Spruce Streets

Philadelphia 4, Pennsylvania 


\section{EMERITUS MEMBERS}

Abernathy, Theodore J. 1834 Eye Street, N. W. Washington 6 , D. C.

Abramson, David I. 916 North Oak Park Avenue Oak Park, Illinois

Adams, John M. School of Medicine University of California 405 Hilgard Avenue Los Angeles 24, California

Adams, Raymond Delacy 320 Adams Street Milton, Massachusetts

Adams, Wright Department of Medicine University of Chicago Chicago 37, Illinois

Aggeler, Paul M. 319 Castenada Avenue San Francisco 16, California

Albert, Alexander Mayo Clinic

Rochester, Minnesota

Albright, Fuller

Massachusetts General Hospital

Boston 14, Massachusetts

Alexander, Benjamin

Medical Research Department

Beth Israel Hospital

330 Brookline Avenue

Boston 15, Massachusetts

Alexander, H. L.

52 Maryland Plaza

St. Louis 8, Missouri

Allan, Frank N. 44 Barnstable Road West Newton, Massachusetts

Allen, Edgar V. Mayo Clinic

Rochester, Minnesota

Allen, J. Garrott

Department of Surgery

School of Medicine

Stanford University

Palo Alto, California
Altemeier, W. A.

University of Cincinnati

College of Medicine

Department of Surgery

Cincinnati General Hospital

Cincinnati 29, Ohio

Althausen, Theodore L.

University of California Hospital

San Francisco 22, California

Altschule, Mark D.

McLean Hospital

Waverley, Massachusetts

Alvarez, Walter C.

700 North Michigan Avenue

Chicago, Illinois

Alving, A. S.

Billings Hospital-M 270

Department of Medicine

950 East 59th Street

Chicago 37, Illinois

The Johns Hopkins Hospital

Baltimore 5, Maryland

Angevine, D. Murray

University of Wisconsin

Medical School

Department of Pathology

Madison 6, Wisconsin

Armstrong, S. Howard, Jr.

Office of Medical Education

Cook County Hospital

1825 West Harrison Street

Chicago 12, Illinois

Astwood, E. B.

New England Center Hospital

30 Bennet Street

Boston 11, Massachusetts

Atchley, Dana W.

622 West 168 th Street

New York 32, New York

Aub, Joseph Charles

Massachusetts General Hospital

Fruit Street

Boston, Massachusetts

BAehr, George

110 East 80th Street

New York 21, New York
ANDRus, E. Cowles
Baetjer, Walter A. 1101 St. Paul Street Baltimore 2, Maryland

Bailey, C. Cabell

58 Bristol Road

Wellesley Hills 81 ,

Massachusetts

Baker, Benjamin M., JR.

9 East Chase Street

Baltimore 2, Maryland

Barach, Alvan L.

929 Park Avenue

New York 28, New York

Barker, Paul S.

University Hospital

Ann Arbor, Michigan

Barr, David P.

Health Insurance Plan

625 Madison Avenue

New York 22, New York

Bassett, Samuel H.

Wadsworth Hospital V A C

Los Angeles 25, California

Batterman, Robert C.

2030 Haste Street

Berkeley 4, California

BaUER, Walter

Massachusetts General Hospital

Boston 14, Massachusetts

Baxter, James $\mathrm{H}$.

Section on Metabolism

National Heart Institute

National Institutes of Health

Bethesda 14, Maryland

BAYLEy, ROBERT H.

University of Oklahoma

Hospitals

800 Northeast Thirteenth St.

Oklahoma City 4, Oklahoma

Bean, William B.

Department of Internal Medicine

University Hospitals

Iowa City, Iowa

BEEBE, Richard T.

Albany Medical College

Albany 8, New York

BEECHER, H. K.

Massachusetts General Hospital

Boston 14, Massachusetts 
Beeson, Paul B.

Department of Internal Medicine

Yale University School of Medicine

333 Cedar Street

New Haven 11, Connecticut

Behnke, A. R. Captain MC USN (Ret.)

1835 Vallejo Street

San Francisco 23, California

Bennett, Granville A.

Office of the Dean

University of Illinois

College of Medicine

1853 West Polk Street

Chicago 12, Illinois

Bennett, H. Stanley

Department of Anatomy

School of Medicine

University of Washington

Seattle 5, Washington

Berry, George P.

Dean

Harvard Medical School

25 Shattuck Street

Boston 15, Massachusetts

Best, Capt. Charles H., Surgeon

$\mathrm{R} C \mathrm{~N}$ V R

University of Toronto

Toronto, Canada

BING, RichaRd J.

Wayne State University Medical School

Detroit, Michigan

Binger, Carl A. L.

21 Lowell Street

Cambridge 38, Massachusetts

Blalock, Alfred

The Johns Hopkins Hospital

Baltimore 5, Maryland

Bland, Edward F.

Massachusetts General Hospital

Boston 14, Massachusetts

BLOCH, Robert G.

Montefiore Hospital

210th Street \& Gun Hill Road

New York 67, New York

Bloomfield, Arthur L.

245 Locust Street

San Francisco 18, California
Bloom Field, RichaRd A. 144 Commonwealth Avenue Boston 16, Massachusetts

Blumgart, Herrman L. 330 Brookline Avenue Boston, Massachusetts

Bock, A. V.

Prospect Hill Road

Harvard, Massachusetts

BordLEy, JAMES, III

Mary Imogene Bassett Hospital

Cooperstown, New York

Bradley, Stanley E.

Presbyterian Hospital

620 West 168th Street

New York 32, New York

Brobeck, JoHN R.

Department of Physiology

University of Pennsylvania

School of Medicine

Philadelphia 4, Pennsylvania

BROH-Kahn, Robert H.

45 Sawmill River Road

Yonkers, New York

Broun, G. O.

1325 S. Grand Boulevard

St. Louis 4, Missouri

Brow, George R.

1390 Sherbrooke St. W., Ste. 5

Montreal, Quebec, Canada

Brown, Charles L. Dean

591 Montgomery Street

Jersey City 2, New Jersey

Brown, JoHN W.

52 Oak Drive

Orinda, California

Brown, Thomas McP.

814-26th Place South

Arlington 2, Virginia

Brown, W. Hurst

280 Bloor Street, West

Toronto 5, Ontario, Canada

Browne, John S. L.

Chairman, Department of Investigative Medicine

Room 106, Medical Building

McGill University

Montreal, P. Q., Canada
Brues, Austin M.

Director, Biology Division

Argonne National Laboratory

P. O. Box 299

Lemont, Illinois

Bukantz, Samuel Charles

Washington University

School of Medicine

600 South Kingshighway

St. Louis 10, Missouri

Bulger, Harold A.

Barnes Hospital

600 South Kingshighway

St. Louis 10, Missouri

Burch, George E.

Department of Medicine

1430 Tulane Avenue

New Orleans 13, Louisiana

Burchenal, Joseph $\mathrm{H}$.

Juniper Hill Road

Noroton, Connecticut

Burnett, Charles $\mathrm{H}$.

School of Medicine

University of North Carolina

Chapel Hill, North Carolina

Burwell, C. Sidney

25 Shattuck Street

Boston 15, Massachusetts

Butler, Allan M.

Massachusetts General Hospital

Boston 14, Massachusetts

Butt, Hugh R.

The Mayo Clinic

102 Second Avenue, S. W.

Rochester, Minnesota

Calmoun, John A., JR.

Medical Director

American Viscose Corporation

1617 Pennsylvania Boulevard

Philadelphia 3, Pennsylvania

Campbell, Walter R.

730 Medical Arts Building

Toronto, Canada

Capps, Joseph A.

1215 East 56th Street

Chicago 37, Illinois

CAPPS, Richard B.

21 Woodley Road

Winnetka, Illinois

Castle, W. B.

Boston City Hospital

Boston 18, Massachusetts 
Caughey, John L., JR.

School of Medicine

Western Reserve University

Cleveland 6, Ohio

Cayer, David

Bowman Gray School of Medicine

Department of Internal Medicine

Winston-Salem 7, North

Carolina

Cecil, Russell L. 449 East 68th Street

New York 21, New York

Chapman, William P.

Massachusetts General Hospital

Boston 14, Massachusetts

Chasis, Herbert

44 East 67th Street

New York, New York

Chesney, Alan M.

The Johns Hopkins University

School of Medicine

Baltimore 5, Maryland

Christie, Ronald

Physician-in-Chief

Royal Victoria Hospital

Montreal 2, Canada

Churchill, Edward D.

Massachusetts General Hospital

Boston 14, Massachusetts

Cleghorn, R. A.

Department of Psychiatry

McGill University

Montreal, P. Q., Canada

Clough, Paul W.

24 East Eager Street

Baltimore 2, Maryland

Cobb, Stanley

34 Fernald Drive

Cambridge 38, Massachusetts

Coburn, Alvin F.

14 Ludlow Drive

Chappaqua, New York

Code, Charles F.

The Mayo Clinic and Mayo Foundation

Rochester, Minnesota

Cogan, David G.

30 Clark Street

Belmont 78, Massachusetts
Coggeshall, Lowell $T$. 5801 South Dorchester Avenue Chicago 37, Illinois

Cohen, Mandel E. Massachusetts General Hospital Boston 14, Massachusetts

Cole, Rufus

Cohomong Wood

Mt. Kisco, New York

Comroe, Julius Hiram, Jr.

555 Laurent Road

Hillsborough, California

Conn, Jerome W.

University Hospital

Ann Arbor, Michigan

Cooke, Robert A.

60 E. 58th Street

New York 22, New York

Cope, Oliver

Massachusetts General Hospital

Boston 14, Massachusetts

Corcoran, Arthur C.

2668 Berkshire Road

Cleveland Heights 6, Ohio

Crawford, John Hamilton

178 Eighth Avenue

Brooklyn 15, New York

Culbertson, James Workman

Department of Internal Medicine

University of Tennessee

858 Madison Avenue

Memphis 3, Tennessee

Curnen, Edward C.

University of North Carolina

School of Medicine

Chapel Hill, North Carolina

Curtis, Arthur C.

University Medical Center

Ann Arbor, Michigan

Curtis, George M.

Kinsman Hall

Ohio State University

Columbus, Ohio

Dameshek, William

205 Beacon Street

Boston 16, Massachusetts

Dammin, Gustave J.

Peter Bent Brigham Hospital

Boston 15, Massachusetts
Darby, William J.

Vanderbilt University

School of Medicine

Department of Medicine

Nashville 4, Tennessee

Darling, Robert C.

157 Glenwood Avenue

Leonia, New Jersey

Darrow, Daniel C.

5847 Howe Drive

Mission, Kansas

Dauphinee, James A.

35 Ava Road

Toronto, Ontario, Canada

Davidson, Charles S.

Thorndike Memorial Laboratory

Boston City Hospital

Boston 18, Massachusetts

Davis, Bernard D.

Department of Bacteriology

Harvard Medical School

Boston 15, Massachusetts

Davis, David

Suite 33

416 Marlborough Street

Boston, Massachusetts

Davis, Jay Conger

529 Medical Arts Building

Minneapolis 2, Minnesota

Davis, William A.

270 Ridgewood Avenue

Glen Ridge, New Jersey

Davison, Wilburt C.

Duke University

Medical School

Box 3701

Durham, North Carolina

Degowin, Elmer L.

Department of Internal Medicine

University Hospitals

Iowa City, Iowa

DeGraff, Arthur C.

850 Park Avenue

New York 21, New York

Derick, Clifford L.

Peter Bent Brigham Hospital

Boston, Massachusetts

Detweiler, H. K.

1007 Medical Arts Building

Toronto, Canada 
Dexter, Lewis

Peter Bent Brigham Hospital

721 Huntington Avenue

Boston 15, Massachusetts

Diamond, Louis K.

Children's Hospital

300 Longwood Avenue

Boston 15, Massachusetts

Dieuaide, Francis R.

300 East 57 th Street

New York 22, New York

Dingle, John Holmes

Department of Preventive Medicine

School of Medicine

Western Reserve University

Cleveland 6, Ohio

Doan, Charles A.

4935 Olentangy Boulevard

Columbus, Ohio

Dobyns, BRown M.

Department of Surgery

Metropolitan General Hospital

Cleveland 9, Ohio

Dochez, Alphonse R.

Presbyterian Hospital

620 West 168th Street

New York 32, New York

Dock, William

450 Clarkson Avenue

Brooklyn 3, New York

Dohan, Francis C.

80 East Princeton Road

Bala Cynwyd, Pennsylvania

Dole, Vincent Paul

Rockefeller Institute Hospital

66th Street \& York Avenue

New York 21, New York

Dorst, Stanley E.

Knoll Road-Amberley

Cincinnati 37, Ohio

Doupe, Joseph

Department of Physiology

University of Manitoba

Medical College

Winnipeg, Canada

Dowling, Harry Filmore

University of Illinois

840 S. Wood Street

Chicago 12, Illinois

Draper, George

840 Park Avenue

New York 21, New York
DRIPPS, ROBERT D.

University of Pennsylvania Hospital

Philadelphia 4, Pennyslvania

EAgLe, Harry

Chief, Laboratory of Cell Biology

National Institute of Allergy and Infectious Diseases

National Institutes of Health

Bethesda 14, Maryland

EArle, David P., JR.

Medical School

Northwestern University

303 East Chicago Avenue

Chicago 11, Illinois

Ebert, Richard V.

6 Virginia Lane

Little Rock, Arkansas

Ebert, Robert H.

Department of Medicine

University Hospitals

2065 Adelbert Road

Cleveland 6, Ohio

Eggleston, Cary

215 East 72nd Street

New York 21, New York

Eichna, Ludwig W.

New York University

College of Medicine

550 . First Avenue

New York 16, New York

Elkinton, J. Russell

Department of Medicine

Hospital of the University of Pennsylvania

3600 Spruce Street

Philadelphia 4, Pennsylvania

Ellis, Laurence B.

319 Longwood Avenue

Boston, Massachusetts

Elsom, Katharine O'Shea

Hospital of University of

Pennsylvania

36th and Spruce Streets

Philadelphia 4, Pennsylvania

Elsom, Kendall A.

Hospital of University of

Pennsylvania

36th and Spruce Streets

Philadelphia 4, Pennsylvania
Emerson, Charles P.

29 Sheridan Road

Wellesley Hills 82,

Massachusetts

Emerson, Kendall, JR.

Peter Bent Brigham Hospital

Boston 15, Massachusetts

ENgel, Frank L.

Duke University

School of Medicine

Durham, North Carolina

Engel, George L.

Strong Memorial Hospital

Rochester, New York

Eppinger, Eugene C.

517 Hammond Street

Chestnut Hill 67, Massachusetts

Epstein, Albert A.

Stebbins Road

Carmel, New York

Ernstene, A. Carlton

Cleveland Clinic

2020 East 93d Street

Cleveland 6, Ohio

Evans, Robert S

Veterans Administration

Hospital

4435 Beacon Avenue

Seattle 8, Washington

Evelyn, Kenneth A.

G. F. Strong Laboratory for Medical Research

Tenth and Heather

Vancouver 9, B. C., Canada

Farquharson, RAy F.

3 Hillhurst Blvd.

Toronto, Canada

FARR, LeE E.

Chairman, Medical Department

Brookhaven National

Laboratory

Upton, Long Island, New York

Fashena, Gladys J.

11550 Wander Lane

Dallas 30, Texas

FaulKner, James M.

Medical Director

Mass Institute of Technology

77 Massachusetts Avenue

Cambridge 39, Massachusetts 
Feldman, Harry A.

State University of New York

College of Medicine

766 Irving Avenue

Syracuse 10, New York

Feller, Alto E.

Department of Microbiology

University of Virginia

Charlottesville, Virginia

Ferrebee, Joseph W.

The Mary Imogene Bassett

Hospital

Cooperstown, New York

Field, Henry, JR.

Veterans Administration Center

Martinsburg, West Virginia

Findley, Thomas

Medical College of Georgia

Augusta, Georgia

Finesinger, JACOB E.

Department of Psychiatry

University of Maryland

School of Medicine

Baltimore 1, Maryland

Finland, Maxwell

Thorndike Memorial

Laboratory

Boston City Hospital

Boston 18, Massachusetts

Fishberg, Arthur M.

1136 Fifth Avenue

New York 28, New York

Fitz-Hugh, Thomas, Jr.

2016 Delancey Place

Philadelphia 3, Pennsylvania

Fleming, William L.

School of Medicine

University of North Carolina

Chapel Hill, North Carolina

Fletcher, A. A.

14 Wychwood Park

Toronto 10, Ontario, Canada

Flink, Edmund B.

5665 Woodlawn Blvd.

Minneapolis 17, Minnestota

Forbes, ANne P.

Massachusetts General Hospital

Fruit Street

Boston 14, Massachusetts

Forkner, Claude E.

35 East 69th Street

New York 21, New York
Fouts, Paul J.

623 Hume Mansur Building

Indianapolis 8, Indiana

Fowler, Willis M.

Department of Internal Medicine

University Hospitals

Iowa City, Iowa

Francis, Thomas, Jr.

University of Michigan

School of Public Health

Ann Arbor, Michigan

Freedberg, A. Stone

Beth Israel Hospital

330 Brookline Avenue

Boston 15, Massachusetts

Freeman, Norman E.

490 Post Street

San Francisco, California

FrEIs, EdWARd D.

Veterans Administration Hospital

2650 Wisconsin Avenue, N. W.

Washington 7, D. C.

Fremont-Smith, Frank

c/o Josiah Macy, Jr.

Foundation

16 West 46th Street

New York 36, New York

FREYBERG, RichaRd $\mathrm{H}$.

535 East 70th Street

New York 21, New York

Friedgood, Harry B.

Director of Medical Education and Research

San Diego County General Hospital

San Diego, California

Friedman, Benjamin

Veterans Administration Hospital

Dallas, Texas

Friedman, Meyer

Harold Brunn Institute

Mount Zion Hospital

1600 Divisadero

San Francisco 15, California

Frisch, A. W.

University of Oregon Medical School

Department of Bacteriology

3181 S. W. Marquam Hill Road

Portland 1, Oregon
Fulton, Frank T.

273 Bowen Street

Providence, Rhode Island

Fulton, Marshall N.

124 Waterman Street

Providence, Rhode Island

Futcher, Palmer H.

Department of Medicine

Johns Hopkins Hospital

Baltimore 5, Maryland

Galdston, Morton

Third N. Y. U. Medical

Division

Goldwater Memorial Hospital

Welfare Island

New York 17, New York

Gamble, C. J.

255 Adams Street

Milton 86, Massachusetts

Gammon, George D.

3400 Spruce Street

University of Pennsylvania

Philadelphia 4, Pennsylvania

Gargill, Samuel L.

474 Beacon Street

Boston 15, Massachusetts

Geiger, Arthur J.

309 Edwards Street

New Haven, Connecticut

Gellhorn, Alfred

Department of Medicine

College of Physicians \&

Surgeons

630 West 168th Street

New York 32, New York

Gibson, John G., II

Department of Medicine

Harvard Medical School

25 Shattuck Street

Boston 15, Massachusetts

Giffin, Herbert $Z$.

1447 Damon Court

Rochester, Minnesota

Gildea, Edwin F.

Department of Psychiatry \& Neurology

Washington University Medical School

Renard Hospital

4940 Audubon Avenue

St. Louis 10, Missouri 
Goldhamer, Stanley M. 10515 Carnegie Avenue Cleveland 6, Ohio

Goldsmith, Grace A. Department of Medicine Tulane University 1430 Tulane Avenue New Orleans 12, Louisiana

Goodhart, Roberts S.

The National Vitamin Foundation, Incorporated

149 East 78th Street

New York 21, New York

Gordon, Burgess L. 4902 Constitution Avenue, N. E. Albuquerque, New Mexico

Gordon, Edgar S. 1300 University Avenue Madison 6, Wisconsin

Gordon, Harry $\mathrm{H}$.

Department of Pediatrics Sinai Hospital of Baltimore Baltimore 15, Maryland

Gorham, L. Whittington 200 East 66th Street New York 21, New York

Grabfield, G. Philip 15 Hutchinson Street Milton 87, Massachusetts

Graham, Duncan 343 Lytton Blvd. Toronto 12, Ontario, Canada

Gray, Horace

122 Olive Mill Lane

Santa Barbara, California

Gray, Seymour J.

Peter Bent Brigham Hospital

721 Huntington Avenue

Boston 15, Massachusetts

Green, Robert Holt

Post Road

RFD 4

Madison, Connecticut

Greene, Carl H. 401 Clinton Avenue Brooklyn 38, New York

Greene, James A.

Room 310, Herman Prof. Bldg. Texas Medical Center Houston 25, Texas
Griffith, J. Q., JR. 2518 West Harold Street Philadelphia 32, Pennsylvania

Grimson, Keith S.

Duke University

School of Medicine

Durham, North Carolina

Gutman, Alexander B.

Mt. Sinai Hospital

100th Street at Fifth Avenue

New York 29, New York

Ham, George C.

Department of Psychiatry

University of North Carolina

Medical School

Chapel Hill, North Carolina

Ham, Thomas Hale

Western Reserve University

School of Medicine

2109 Adelbert Road

Cleveland 6, Ohio

Hamblen, Edwin Crowell

Duke Hospital

Durham, North Carolina

Hamburger, Morton Cincinnati General Hospital Cincinnati 29, Ohio

Hamilton, Bengt

8311 Drexel Avenue

Chicago 19, Illinois

Hanger, Franklin M.

Presbyterian Hospital

168th Street \& Broadway

New York 32, New York

Hansen, Arild E.

Bruce Lyon Memorial Research Laboratory

Children's Hospital of the East Bay

Oakland 9, California

Harford, Carl G.

Barnes Hospital

600 S. Kingshighway

St. Louis 10, Missouri

Harrell, George T.

College of Medicine

University of Florida

Gainesville, Florida

Harris, Jerome S.

Duke Hospital

Durham, North Carolina
Harrison, Harold E.

3001 Fordney Lane

Baltimore 7, Maryland

Harrison, Tinsley $R$.

Medical College of Alabama

Department of Medicine

Birmingham 3, Alabama

Harvey, A. McGehee

Johns Hopkins Hospital

Baltimore 5, Maryland

Havens, W. Paul, Jr.

1025 Walnut Street

Philadelphia, Pennsylvania

Hayman, Joseph M., JR.

Tufts University School of Medicine

136 Harrison Avenue

Boston 11, Massachusetts

Heath, Clark W.

18 Latin Way

Tufts University

Medford 55, Massachusetts

Hecht, Hans $H$.

Department of Internal Medicine

Salt Lake General Hospital

175 East 21st South St.

Salt Lake City 12, Utah

Heinle, Robert W.

Research Division

The Upjohn Company

Kalamazoo, Michigan

Heller, Carl G.

Pacific Northwest Research Foundation, Inc.

1122 Columbia Street

Seattle 4, Washington

Hem pelmanN, Louis H., JR.

Strong Memorial Hospital

Rochester, New York

Hench, Philip S.

517-4th Street, S. W.

Rochester, Minnesota

Herrell, Wallace E.

Lexington Clinic

1221 South Broadway

Lexington, Kentucky

Herrmann, George R.

The John Sealy Hospital

University of Texas Medical

School

816 Avenue B

Galveston, Texas 
Hines, Edgar A., JR. Mayo Clinic

102-110 Second Avenue, SW

Rochester, Minnesota

Hinshaw, H. Corwin

8 Crest Road

Belvedere, California

Hirsch, Edwin F.

Presbyterian-St. Luke's Hospital

1753 West Congress Parkway

Chicago 5, Illinois

Hirst, George K.

The Public Health Research Institute of the City of New York, Incorporated

Foot of East 15th Street

New York 9, New York

Hoffbauer, Frederick W. 4701 East 34th Street

Minneapolis 6, Minnesota

Holt, Emmett L., JR.

Bellevue Hospital

Department of Pediatrics, G-6

First Avenue at 26th Street

New York 16, New York

Hoobler, Sibley W.

2228 Belmont Road

Ann Arbor, Michigan

Horsfall, Frank L., JR.

Rockefeller Institute

New York 21, New York

Horstmann, Dorothy M.

Section of Epidemiology and Preventive Medicine

Yale University School of Medicine

333 Cedar Street

New Haven 11, Connecticut

Horton, Bayard T.

Mayo Clinic

Rochester, Minnesota

Hunter, Thomas $\mathrm{H}$.

Office of the Dean

University of Virginia

School of Medicine

Charlottesville, Virginia

Hurxthal, Lewis M.

605 Commonwealth Avenue

Boston, Massachusetts
INGELFINGER, FRANZ J.

Evans Memorial Hospital

65 East Newton Street

Boston 18, Massachusetts

ISAACS, RAPHAEL

104 S. Michigan Avenue

Chicago 3, Illinois

JaCKson, Henry, Jr.

Thorndike Memorial Laboratory

Boston City Hospital

Boston, Massachusetts

Jacobson, Bernard M.

275 Charles Street

Boston 14, Massachusetts

Jacobson, Leon O.

University of Chicago

950 East 59th Street

Chicago 37, Illinois

Jacox, Ralph FrankLiN

142 Greenaway Road

Rochester, New York

JAGER, B. V.

Veterans Administration

Hospital

Irving Avenue \& University Place

Syracuse 10, New York

JaILer, Joseph William

Department of Medicine

College of Physicians \& Surgeons

Columbia University

620 West 168th Street

New York 32, New York

Janeway, Charles A.

Children's Hospital

300 Longwood Avenue

Boston 15, Massachusetts

JefFers, William A.

University Hospital

Philadelphia 4, Pennsylvania

Jeghers, Harold J.

Seton Hall College of Medicine

Jersey City Medical Center

Jersey City 4, New Jersey

JOHnson, ROBERT E.

804 West Green Street

Urbana, Illinois

Johnston, Franklin D.

Heart Station

University Hospital

Ann Arbor, Michigan
Jolliffe, Norman

910 Park Avenue

New York 21, New York

Jones, Chester M.

Room 1004, Warren Building

275 Charles Street

Boston 14, Massachusetts

Josephs, H. W.

Johns Hopkins Hospital

Baltimore, Maryland

Joslin, Elliott P.

15 Joslin Road

Boston 15, Massachusetts

Kaltreider, N. L.

260 Crittenden Boulevard

Rochester 20, New York

Kark, Robert M.

University of Illinois

College of Medicine

1853 West Polk Street

Chicago 12, Illinois

Karnofsky, David A.

45 Larchmont Avenue

Larchmont, New York

Katz, Louis N.

Director, Cardiovascular

Department

Michael Reese Hospital

Chicago 16, Illinois

Kay, Calvin F.

Hospital of the University of Pennsylvania

Philadelphia 4, Pennsylvania

Keating, F. R., JR. 200 First Street, S. W.

Rochester, Minnesota

Keefer, Chester S.

Evans Memorial

65 East Newton Street

Boston 18, Massachusetts

Keith, Norman M.

Mayo Clinic

Rochester, Minnesota

Kelley, William Henry

215 East Jackson Street

Orlando, Florida

Kennedy, J. Allen

919 Bennie-Dillon Building

Nashville 3, Tennessee 
Kenyon, A. T.

950 E. 59th Street

Chicago, Illinois

Kerby, Grace P.

Duke University School of Medicine

Box 3328

Durham, North Carolina

Kern, Richard A. 1239 Remington Road

Wynnewood, Pennsylvania

King, JoHn T.

1210 Eutaw Place

Baltimore 10, Maryland

Kinsella, Ralph A. 4954 Lindell Boulevard St. Louis 8, Missouri

Kinsman, J. Murray 2415 Longest Avenue Louisville 4, Kentucky

Kirsner, Joseph B. 5807 Dorchester Avenue Chicago, Illinois

Klumpe, Theonore G. 1450 Broadway New York 18, New York

Kneeland, Yale, Jr. 1010 Fifth Avenue New York 28, New York

Kornberg, Arthur Department of Biochemistry Stanford University School of Medicine

Palo Alto, California

Korns, Horace M.

Dey Building

Iowa City, Iowa

Kossmann, Charles E. N. Y. U. College of Medicine 550 First Avenue

New York 16, New York

Kountz, William B.

Washington University School of Medicine

5600 Arsenal Street

St. Louis 9, Missouri

Kramer, Benjamin

4802 Tenth Avenue

Brooklyn 19, New York
KrumbhaAr, Edward B.

Box 4056

Philadelphia 18, Pennsylvania

Kydd, David M.

Department of Internal Medicine

State University of New York

451 Clarkson Avenue

Brooklyn 3, New York

Landis, Eugene M.

Dept. of Physiology

Harvard Medical School

25 Shattuck Street

Boston, Massachusetts

LAndowne, Milton

Levindale

Baltimore 15, Maryland

Langmuir, Alexander D.

Communicable Disease Center

50 Seventh Street, N. E.

Atlanta 23, Georgia

Lauson, Henry O.

Albert Einstein College of Medicine

Eastchester Road \& Morris Park Avenue

New York 61, New York

Lavietes, Paul $\mathrm{H}$.

181 Alston Avenue

New Haven 15, Connecticut

Lawrence, J. H.

Donner Laboratory of Medical Physics

University of California

Berkeley 4, California

LAWRENCE, JOHN S.

2090 Stradella Road

Los Angeles 24, California

Lee, Roger I.

264 Beacon Street

Boston, Massachusetts

LEITER, LOUIS

Montefiore Hospital

210th Street \& Bainbridge Avenue

Bronx 67, New York

LERMAN, JACOB

370 Commonwealth Avenue

Boston, Massachusetts
Levine, Samuel A.

270 Commonwealth Avenue

Boston 16, Massachusetts

Levy, Robert L.

730 Park Avenue

New York 21, New York

Lloyd, Charles Wait

State University of New York

Medical Center at Syracuse

Syracuse, New York

Locke, Edwin A.

Wilton, New Hampshire

Loeb, Robert F.

950 Park Avenue

New York 28, New York

LOEBEL, Robert O.

205 East 78th Street, Sta. Y

New York 21, New York

Long, C. N. Hugh

Department of Physiology

Yale University

New Haven 11, Connecticut

Long, Perrin H.

Department of Medicine

450 Clarkson Avenue

Brooklyn 3, New York

Loosli, Clayton G.

University of Southern California

2025 Zonal Avenue

Los Angeles 33, California

Lowell, Francis C.

Massachusetts General Hospital

Fruit Street

Boston 14, Massachusetts

Luetscher, John A., JR.

Stanford-Palo Alto Medical Center

304 Pasteur Drive

Palo Alto, California

Lukens, Francis D. W.

Maloney Clinic, Room 809

University of Pennsylvania

Philadelphia 4, Pennsylvania

Lyons, Richard H.

R. F. D. 1

Fayetteville, New York

MacBryde, Cyril M.

105 Clayprice Building

9216 Clayton Road

St. Louis 24, Missouri 
Machella, Thomas E.

University Hospital

3400 Spruce Street

Philadelphia 4, Pennsylvania

Mackay, Eaton M.

309 W. 8th Street

Santa Ana, California

MacLeod, A. Garrard

Special Publications Department

The Upjohn Company

Kalamazoo, Michigan

MacLeod, Colin M.

Hospital of the University of Pennsylvania

Philadelphia 4, Pennsylvania

Magnuson, Harold J. 6012-28th Street, N. W.

Washington $15, \mathrm{D}$. C.

MAJOR, RALPH

University of Kansas

School of Medicine

Kansas City, Kansas

Marble, Alexander

131 Laurel Road

Chestnut Hill 67, Massachusetts

Martin, Lay

1201 N. Calvert St.

Baltimore 2, Maryland

Marvin, Harold M.

303 Whitney Avenue

New Haven 11, Connecticut

Mason, Edward H.

452 Stanstead Avenue

Town of Mt. Royal

P. Q., Canada

McCann, Wm. S.

University of Rochester

School of Medicine

Rochester 20, New York

McCarty, Maclyn

The Rockefeller Institute

66th Street \& York Avenue

New York 21, New York

McClellan, Walter Stuart

406 Whitehead Circle

Chapel Hill, North Carolina

McEwen, Currier

550 First Avenue

New York 16, New York
MCDERMotT, WaLSH

New York Hospital

525 East 68th Street

New York 21, New York

McIntosh, John F.

19 Springfield Avenue

Westmount 6, Quebec, Canada

McIntosh, Rustin

630 West 168th Street

New York 32, New York

McIver, Monroe A.

Mary Imogene Bassett Hospital

Cooperstown, New York

McKhann, Charles F., Jr.

Babies \& Childrens Hospital

University Hospitals

Cleveland, Ohio

McLean, Franklin C.

5545 University Avenue

Chicago 37, Illinois

McQuarrie, Irvine

Director of Research

Children's Hospital

Oakland 9, California

Meakins, J. C.

University Clinic

Royal Victoria Hospital

Montreal, Quebec, Canada

Means, James $\mathrm{H}$.

60 Mount Vernon Street

Boston 8, Massachusetts

Meiklejohn, Gordon

University of Colorado Medical Center

4200 East Ninth Avenue

Denver 20, Colorado

Mendlowitz, Milton

2 East 95th Street

New York 28, New York

Merrill, Arthur J.

35 Fourth Street, N. E.

Atlanta 8, Georgia

Merritt, H. Houston

Neurological Institute

710 West 168th Street

New York 32, New York

Mettier, Stacy R.

University of California

Medical School

San Francisco 22, California
Meyer, Ovid O.

State of Wisconsin General

Hospital

Madison, Wisconsin

Middleton, William S.

4200 Cathedral Avenue, N. W.

Washington 16, D. C.

Milhorat, Ade Thomas

515 East 71st Street

New York 21, New York

Miller, Benjamin F.

May Institute for Medical

Research

421 Ridgeway Avenue

Cincinnati 24, Ohio

Miller, C. Phillip, JR.

Department of Medicine

University of Chicago

Chicago 37, Illinois

Miller, David K.

E. J. Meyer Memorial Hospital

462 Grider Street

Buffalo 15, New York

Miller, Franklin R.

Jefferson Medical College and Hospital

Division of Hematology

Philadelphia 7, Pennsylvania

Miller, Max

Lakeside Hospital

2065 Adelbert Road

Cleveland 6, Ohio

MilleR, T. Grier

133 South 36th Street

Philadelphia 4, Pennsylvania

Mirick, George S.

Baltimore City Hospitals

Baltimore 24, Maryland

Mirsky, I. Arthur

Department of Clinical Science

University of Pittsburgh

School of Medicine

3811 O'Hara Street

Pittsburgh 13, Pennsylvania

Montgomery, Hugh

119 Glenn Road

Ardmore, Pennsylvania 
Moore, Carl V.

Department of Internal Medicine

Washington University

St. Louis 12, Missouri

Moore, Francis D.

Peter Bent Brigham Hospital

721 Huntington Avenue

Boston 15, Massachusetts

Morgan, Hugh J.

15 White Bridge Road

Nashville 5, Tennessee

Morton, JoHn J.

1915 Westfall Road

Rochester 18, New York

Mosenthal, H. O.

889 Lexington Avenue

New York 21, New York

Most, HarRy

New York University

College of Medicine

550 First Avenue

New York 16, New York

Moyer, Carl A.

Department of Surgery

Washington University

School of Medicine

St. Louis 10, Missouri

Muckenfuss, Ralph S.

Naval Medical Research Institute

Bethesda 14, Maryland

Murphy, William Parry

20 Gloucester Street

Boston 15, Massachusetts

Myers, Gordon B.

789 Westchester

Grosse Pointe Park 30,

Michigan

Myers, Jack D.

Department of Medicine

University of Pittsburgh

School of Medicine

Pittsburgh 13, Pennsylvania

NAJjar, Victor A.

Department of Microbiology

Vanderbilt University

School of Medicine

Nashville 5, Tennessee
Neefe, John R.

St. Petersburg Medical Clinic

501-11th Street, North

St. Petersburg, Florida

Newman, Elliot Voss

Department of Experimental Medicine

Vanderbilt University Hospital

Nashville 5, Tennessee

Nicholson, W. M.

Department of Medicine

Duke University

School of Medicine

Durham, North Carolina

O'Hare, James P.

Peter Bent Brigham Hospital

721 Huntington Avenue

Boston, Massachusetts

Ordway, Thomas

161 South Lake Avenue

Albany, New York

Osgood, Edwin E.

University of Oregon Medical School

3181 S. W. Sam Jackson Park Road

Portland 1, Oregon

OtTENBERG, Reuben

1112 Park Avenue

New York, New York

Palmer, Robert S.

61 Griggs Road

Brookline, Massachusetts

Palmer, Walter L.

1320 East 58th Street

Chicago 37, Illinois

Pardee, Harold E. B.

772 Park Avenue

New York 21, New York

Park, Edwards A.

Johns Hopkins Hospital

Department of Pathology

Baltimore 5, Maryland

Parker, Robert F.

2109 Adelbert Road

Cleveland 6, Ohio

Parson, William

University of Virginia

School of Medicine

Charlottesville, Virginia
Patek, Arthur J., Jr.

755 Park Avenue

New York 21, New York

Patterson, John L., Jr.

Department of Medicine

Medical College of Virginia

Richmond, Virginia

Paul, John R.

Yale University

Section of Epidemiology and

Preventive Medicine

333 Cedar Street

New Haven 11, Connecticut

Pearson, Olof H.

444 East 68th Street

New York 21, New York

Pepper, O. H. Perry

945 Parkes Run Lane

Villanova, Pennsylvania

Perera, George A.

622 West 168th Street

New York 32, New York

Pitts, Robert F.

Cornell University Medical

College

1300 York Avenue

New York 21, New York

Pollack, Herbert

70 E. 77th Street

New York 21, New York

Post, Joseph

1100 Madison Avenue

New York 28, New York

Powers, Grover F.

New Haven Hospital

New Haven 4, Connecticut

Pratt, Edward L.

Department of Pediatrics

Southwestern Medical School

5323 Harry Hines Blvd.

Dallas 35, Texas

Prinzmetal, Myron

810 Rexford

Beverly Hills, California

Pritchard, Walter $H$.

Lakeside Hospital

Cleveland 6, Ohio 
Proger, Samuel H.

New England Center Hospital

171 Harrison Avenue

Boston 11, Massachusetts

Putnam, Tracy Jackson

450 N. Bedford Drive

Beverly Hills, California

Rackemann, Francis M.

263 Beacon Street

Boston 16, Massachusetts

Ragan, Charles

620 West 168th Street

New York 32, New York

Rammelkamp, Charles H.

Department of Research Laboratories

Cleveland Metropolitan General Hospital

3395 Scranton Road

Cleveland 9, Ohio

Rantz, Lowell A.

Stanford Medical Center

300 Pasteur Drive

Palo Alto, California

Rapoport, Samuel

Kuckhoff Strasse 45

Berlin-Niederschonhausen

Germany

Rasmussen, Aaron Frederick, JR.

Department of Infectious Diseases

U. C. L. A. Medical School

Los Angeles 24, California

Rawson, Rulon W.

Memorial Hospital

444 East 68th Street

New York 21, New York

Reifenstein, Edward C., Jr.

50 Hoot Owl Terrace

Smoke Rise

Butler, New Jersey

Reimann, Hobart A.

Shiraz Medical Center

Shiraz, Iran

Reznikoff, Paul

New York Hospital

525 East 68th Street

New York 21, New York
Richards, Dickinson W.

620 West 168th Street

New York 32, New York

Richardson, Henry B.

4 East 89th Street

New York 28, New York

Ricketts, Henry T.

Department of Medicine

University of Chicago

950 East 59th Street

Chicago 37, Illinois

Riecker, Herman H.

326 N. Ingalls Street

Ann Arbor, Michigan

Riley, Richard L.

1901 Dixon Road

Baltimore 9, Maryland

Rinehart, James F.

University of California

School of Medicine

San Francisco 22, California

Riseman, Joseph E. F.

330 Brookline Avenue

Boston, Massachusetts

Rivers, Thomas M.

Rockefeller Hospital

66th Street \& York Avenue

New York 21, New York

Robertson, O. H.

9150 Los Gatos Highway

Santa Cruz, California

Robinson, G. Canby

320 Carpenter Street

Greenport, New York

ROBINSON, HaRry J.

Merck Institute for Therapeutic

Research

Rahway, New Jersey

Romano, John

Strong Memorial Hospital

Rochester 20, New York

Root, Howard F.

44 Dwight Street

Brookline, Massachusetts

Ropes, Marian W.

Massachusetts General Hospital

Boston 14, Massachusetts
Rose, Bram

636 Victoria Avenue

Westmount, Quebec, Canada

Rose, HarRy M.

Columbia University

College of Physicians \&

Surgeons

630 W. 168th Street

New York 32, New York

Rosenberg, David H.

$111 \mathrm{~N}$. Wabash Avenue

Chicago 2, Illinois

Rosenberg, Edward F.

Garland Building

Corner Washington \& Wabash

Chicago 2, Illinois

Ross, Joseph F.

Department of Nuclear Medicine \& Radiation Biology

School of Medicine

UCLA Medical Center

Los Angeles 24, California

Rothbard, Sidney

Department of Medicine

New York Hospital

525 East 68th Street

New York 21, New York

Rowntree, Leonard G.

632 DuPont Building

Miami 32, Florida

RUEGSEGgER, J. M.

Lederle Laboratories

Pearl River, New York

Ruffin, Julian M.

Box 3703

Department of Medicine

Duke University

Durham, North Carolina

Rundles, Ralph Wayne

Duke University

School of Medicine

Department of Medicine

Durham, North Carolina

Rutstein, David D.

Harvard Medical School

Department of Preventive Medicine

25 Shattuck Street

Boston 15, Massachusetts 
Rytand, David A.

Stanford Medical Center

300 Pasteur Drive

Palo Alto, California

Sabin, Albert B.

The Children's Hospital Research Foundation

Elland \& Bethesda Avenues

Cincinnati 29, Ohio

SAdusk, Joseph F., JR.

411-30th Street

Oakland 9, California

Salk, Jonas E.

Virus Research Laboratory

Municipal Hospital

Terrace \& Darragh Streets

Pittsburgh 13, Pennsylvania

SCHIfF, LeON

Gastric Laboratory

Cincinnati General Hospital

Cincinnati 29, Ohio

Schloss, O. M.

Address unknown

Schmitz, Henry L. 5844 Stony Island

Chicago 37, Illinois

Schroeder, Henry A.

West Brattleboro, Vermont

Scott, Thomas F. McNair

421 Station Avenue

North Hills, Glenside,

Pennsylvania

Scott, W. J. Merle

Strong Memorial Hospital

Rochester, New York

Sebrell, William H., JR.

Director, Institute of Nutrition Sciences

Columbia University

School of Public Health \& Administrative Medicine

600 West 168th Street

New York 32, New York
Seegal, David

Columbia University Research Service

Goldwater Memorial Hospital

Welfare Island

New York 17, New York

Selye, Hans

Institute of Experimental Medicine and Surgery

University of Montreal

C. P. 6128

Montreal, Canada

Severinghaus, Elmer L.

53 Stonebridge Road

Montclair, New Jersey

Shannon, James A.

The National Institutes of Health

Director

Bethesda 14, Maryland

Shattuck, George C.

450 Warren Street

Brookline, Massachusetts

Shen, Shu Chu

206 Lexington Street

Belmont, Massachusetts

Shibley, G. S.

2469 Kenilworth Road

Cleveland Heights, Ohio

Shipley, Reginald A.

Veterans Administration Hospital

7300 York Road

Cleveland 30, Ohio

Short, Charles L.

264 Beacon Street

Boston, Massachusetts

Slavin, Howard B.

University of Rochester

School of Medicine

260 Crittenden Boulevard

Rochester 20, New York

Smadel, Joseph E.

Public Health Service

National Institutes of Health

Bethesda 14, Maryland
Smith, David Tillerson

Duke Medical School

Durham, North Carolina

Sмith, John R.

Washington University

Department of Internal Medicine

600 South Kingshighway

St. Louis 10, Missouri

Snell, Albert M.

Palo Alto Medical Clinic

300 Homer Avenue

Palo Alto, California

Sodeman, William A.

Jefferson Medical College

1025 Walnut Street

Philadelphia 7, Pennsylvania

SOFFER, Louis J.

1155 Park Avenue

New York 28, New York

Sokolow, Maurice

University of California Medical Center

San Francisco 22, California

Soskin, Samuel

748 South Beverly Glen Boulevard

Los Angeles 24, California

Spies, Tom D.

Nutrition Clinic

Hillman Hospital

1901 Sixth Avenue. South

Birmingham 3, Alabama

Spink, Wesley W.

University Hospital

Minneapolis 14, Minnesota

Sprague, R. G.

Mayo Clinic

Rochester, Minnesota

Stare, Frederick J.

Department of Nutrition

Harvard University

1 Shattuck Street

Boston 15, Massachusetts

StARr, ISAAC

University of Pennsylvania Medical School

Philadelphia 4, Pennsylvania 
Starr, Paul

Box 302

1200 North State Street

Los Angeles 33, California

Stead, E. A., Jr.

2122 Myrtle Drive

Durham, North Carolina

Steele, J. Murray

Goldwater Memorial Hospital

Welfare Island

New York 17, New York

Stewart, Harold J.

New York Hospital

525 East 68th Street

New York 21, New York

Stewart, John D.

Edward J. Meyer Memorial Hospital

462 Grider Street

Buffalo 15, New York

Stillman, Edgar

6 Sutton Square

New York 22, New York

Strauss, Elias

3707 Gaston Avenue

Dallas, Texas

Strauss, Maurice B.

27 Grove Hill Avenue

Newtonville 60, Massachusetts

Strouse, Solomon

405 North Bedford Drive

Beverly Hills, California

Sturgis, Cyrus C.

Simpson Memorial Institute

Ann Arbor, Michigan

Sunderman, F. William

Jefferson Medical College

1025 Walnut Street

Philadelphia 7, Pennsylvania

Sutliff, Whelan Dwight

Veterans Administration

Medical Teaching Group

Hospital (Kennedy)

Memphis 15, Tennessee

Syverton, Jerome T.

Professor \& Head

Department of Bacteriology \& Immunology

University of Minnesota Medical School

Minneapolis 14, Minnesota
TAgER, MORRIS

Department of Bacteriology

Emery University

Woodruff Memorial Building

Atlanta 22, Georgia

Tagnon, Henry J.

Institut Jules Bordet

Rue Heger Bordet

Brussells, Belgium

TALbot, Fritz

24 Cottage Farm Road

Brookline 46, Massachusetts

Talbot, Nathan Bill

Massachusetts General Hospital

Boston 14, Massachusetts

Talbott, John $\mathrm{H}$.

Buffalo General Hospital

100 High Street

Buffalo 3, New York

Thomas, Caroline B.

314 Overhill Road

Baltimore 10, Maryland

Thomas, Lewis

Department of Medicine

N. Y. U. College of Medicine

550 First Avenue

New York 16, New York

ThOMSON, K. JeFFERSON

1 Madison Avenue

New York 10, New York

Thorn, George W.

Peter Bent Brigham Hospital

Boston, Massachusetts

Tillett, William S.

550 First Avenue

New York 16, New York

Tocantins, Leandro M.

Jefferson Medical College

1025 Walnut Street

Philadelphia 7, Pennsylvania

Tompsett, Ralph R.

Baylor University Medical

Center

3500 Gaston Avenue

Dallas 10, Texas

Turner, Joseph C.

620 West 168th Street

New York 32, New York
TURner, Roy $H$.

1430 Tulane Avenue

New Orlean 12, I.a.

Turner, Thomas B.

The Johns Hopkins University

School of Medicine

725 N. Wolfe Street

Baltimore 5, Maryland

VANDER VeER, Joseph B.

330 S. 9th Street

Philadelphia 7, $\mathrm{Pa}$.

Vilter, Richard W.

Cincinnati General Hospital

Cincinnati 29, Ohio

Wakefield, E. G.

Mayo Clinic

Rochester, Minnesota

Walker, Arthur M.

University Club

1135-16th Street

Washington, D. C.

Wallace, William M.

2065 Adelbert Road

Cleveland 6, Ohio

WARD, ROBERT

Children's Hospital

4614 Sunset Boulevard

Los Angeles 27, California

Warren, Stafford L.

School of Medicine

University of California

Los Angeles 24, California

Watkins, Charles $H$.

Mayo Clinic

Rochester, Minnesota

Watson, C. J.

Medicine Department

C-309 Mayo Building

University of Minnesota

Minneapolis 14, Minnesota

WATSON, JANET

235 Adams Street

Brooklyn 1, New York

WATSON, ROBERT F.

New York Hospital

525 East 68th Street

New York 21, New York 
WEARN, J. T.

University Hospitals

Cleveland 6, Ohio

Webster, Bruce

449 East 68th Street

New York 21, New York

Weech, Alexander A.

Children's Hospital Research

Foundation

Elland Avenue \& Bethesda

Cincinnati 29, Ohio

Wegria, Rene

St. Louis University

School of Medicine

1325 South Grand Boulevard

St. Louis 4, Missouri

Weinstein, Louis

26 Greylock Road

Newtonville, Massachusetts

Weisberger, Austin S.

Lakeside Hospital

2065 Adelbert Road

Cleveland 6, Ohio

Welt, Louis G.

Department of Internal Medicine

University of North Carolina

School of Medicine

Chapel Hill, North Carolina

Werner, Sidney C.

The Presbyterian Hospital

622 West 168th Street

New York 32, New York

West, Howard F.

1100 North Mission Road

Los Angeles 33, California

White, James C.

Massachusetts General Hospital

Boston 14, Massachusetts

White, Paul D.

264 Beacon Street

Boston, Massachusetts

Whitten berger, James L.

Harvard School of Public Health

Department of Physiology

55 Shattuck Street

Boston 15, Massachusetts

Wilbur, DWight L.

655 Sutter Street

San Francisco 2, California
Wilder, Russell $M$.

Mayo Clinic

Rochester, Minnesota

WiLkins, R. W.

Evans Memorial Hospital

65 E. Newton Street

Boston 18, Massachusetts

Williams, John R.

380 Monroe Avenue

Rochester 7, New York

Williams, John R., JR. 380 Monroe Avenue

Rochester 7, New York

Williams, Robert H.

University of Washington

School of Medicine

Seattle 5, Washington

Willis, Henry Stuart

North Carolina Sanatorium System

Chapel Hill, North Carolina

Wintrobe, Maxwell M.

175 East 21st South Street

Salt Lake City 15, Utah

Wolf, Stewart G., JR.

University Hospital

800 N. E. 13th Street

Oklahoma City 4, Oklahoma

Wolferth, Charles C.

26 Raynham Road

Merion Station, Pennsylvania

WolfF, Harold G.

Department of Medicine

New York Hospital

525 East 68th Street

New York 21, New York

Wollaeger, ERIC E.

102-110 Second Avenue S. W.

Rochester, Minnesota

Wood, Earl Howard

Mayo Foundation

University of Minnesota

Rochester, Minnesota

Wood, Francis Clark

212 Laurel Lane

Haverford, Pennsylvania

Wood, J. Edwin., JR.

Box 3454

University Station

Charlottesville, Virginia
WoOD, William Barry, JR.

The Johns Hopkins University

School of Medicine

725 North Wolfe Street

Baltimore 5, Maryland

Woodward, Theodore E.

Department of Medicine

University of Maryland Medical School

Baltimore 1, Maryland

Wright, Irving S.

Cornell University Medical College

525 East 68th Street

New York 21, New York

YANNET, HERMAN

Southbury Training School

Southbury, Connecticut

Yater, Wallace M.

1780 Massachusetts Avenue

N. W.

Washington 6, D. C.

Youmans, JoHn B.

Technical Director of Research

U. S. Army Medical Research and Development Command

Main Navy Building

Washington 25, D. C.

Young, Lawrence E.

University of Rochester

School of Medicine

260 Crittenden Boulevard

Rochester 20, New York

Zamecnik, Paul C.

Argilla Road

Ipswich, Massachusetts

Zerfas, L. G.

Route 1

Camby, Indiana

ZIFF, MORRIS

University of Texas

Department of Internal

Medicine

Southwestern Medical School

5323 Harry Hines Boulevard

Dallas 19, Texas

Zubrod, Charles G.

National Cancer Institute

National Institutes of Health

Bethesda 14, Maryland 


\section{DECEASED MEMBERS}

Aвbott, William Osler

ADDIS, THOMAS

ADLER, IsAAC

Amoss, Harold L.

Ashford, Bailey Kelly

Austin, J. Harold

Austrian, Charles R.

Avery, O. T.

Baldridge, Clarence William

Barnett, George D.

Barron, E. S. Guzman

BaUman, Louis

Beckman, William Wood

Bergenstal, Delbert M.

Bethell, Frank $\mathrm{H}$.

Blackfan, Kenneth Daniel

Blake, Francis Gilman

Blankenhorn, Marion A.

BOAS, ERNST P.

Boothby, Walter M.

Brown, George Elgie

Buckman, Thomas Ellowood

Cam PBell, How ard

Chickering, Henry $T$.

Christian, Henry Asbury

Cohn, Alfred E.

Cowie, David Murray

Cutler, Elliott C.

Dawson, Martin H.

Decherd, GEORGe M., JR.

DOBRINER, KONRAD

Drinker, Cecil K.

DuBois, Eugene F.

Ellsworth, Read Mclane

EMERSON, HAVEN

Emery, Edward Stanley, Jr.

Evans, Frank A.

Ferris, Eugene B., Jr.

Fitz, Reginald

Fleischner, Emanuel C.

Foster, Nellis Barnes

Friedenwald, Jonas B.

Gamble, James L.

Geyelin. Henry Rawle

Gilbert, Newell C.
Guthrie, Clyde Graeme

Haden, Russell L.

Hallock, Phillip

Hamburger, Walter Wile

Hamman, Louis Virgil

Harris, Seale, Jr.

Harrop, George Argale, Jr.

Hart, TheOdore S.

Hertz, Saul

Hess, Alfred Fabian

Hewlett, Albion Walter

Hoagland, Charles Lee

Hoover, Charles Franklin

Howard, Alan Campbell P.

HOWLAND, JOHN

Hunt, James Ramsay

Irons, ERNEST E.

Janeway, Theodore Caldwell

Janney, Nelson Wilson

Jones, Willard T.

Kessel, Leo

Koessler, KarL Konrad

Krause, Allen Kramer

LASHMET, Floyd H.

LEMON, WiLlis S.

Libman, EmanUel

LiLIENTHAL, JOSEPH L., JR.

LOCKWOOD, JOHN S.

LONGCOPE, WARFIELD T.

LORD, Frederick TAYLOR

Marriott, William McKim

McEachern, Donald

McLester, JAmes S.

McPhedran, F. Maurice

McPhedran, William F.

McVicar, Charles Stanley

Meltzer, Samuel J.

Minot, George Richards

MOORE, JOSEPH E.

Morris, Roger Sylvester

Moss, William L.

Musser, John Herr, Jr.

Nathanson, Ira T.

Newburgh, Louis $H$.

Nye, Robert N.
Oppenheimer, B. S.

Palmer, Walter Walker

Peabody, Francis Weld

Pemberton, Ralph

Peters, John P.

Pohle, Frederick John

Pratt, Joseph $\mathrm{H}$.

Quinby, William C.

Rhoads, Cornelius P.,

Rosenbaum, Jack Davidson

Rothschild, Marcus A.

SAILER, JOSEPH

Salter, William Thomas

Schoenbach, Emanuel B.

Scott, Roy Webley

Sedgwick, J. P.

Sellards, Andrew Watson

Shelling, David H.

SHORR, EPHRAIM

SMith, Bertnard

Smith, Fred M.

Soley, Mayo Hamilton

Stadie, W. S.

Steele, John Dutton

Stephens, Doran J.

Stillman, Ernest Goodwich

Stone, Willard John

SwIFT, HOMER Fordyce

Thayer, William Sydney

Thompson, Willard O.

Thompson, William P.

Tisdall, Frederick F.

Trask, James Dowling

Turner, KenNeth B.

Wadsworth, Augustus B.

Walker, IsaAC ChandLer

Wallace, George Barclay

WEIL, RichaRd

Weiss, Soma

WeSt, John Robert

West, RaNDOLPH

Williamson, Charles S.

Wilson, Frank Norman

Winkler, Alexander W.

WoOdyatT, R. T. 